\title{
面向高性能工业仿真的交互可视分析引擎
}

\author{
曹轶 $^{1,2)}$ ，王华维 ${ }^{1,2}$ ，夏芳 ${ }^{1,2)}$ ，张哲 ${ }^{2}$ ，艾志玮 ${ }^{1,2)}$ ，吴付坤 ${ }^{2)}$ ，曹斯铭 ${ }^{2}$ \\ ${ }^{1)}$ (北京应用物理与计算数学研究所 北京 100094) \\ 2) (中物院高性能数值模拟软件中心 北京 100088) \\ (cao_yi@iapcm.ac.cn)
}

\begin{abstract}
摘 要: 针对大规模仿真数据对仿真设计分析造成的前后连贯交互力弱、可视交互力低的问题，提出一个面向高性 能工业仿真的交互可视分析引擎. 首先构建可高效耦合几何模型和计算网格的三维混合数据模型; 然后提出面向前 后连贯仿真设计的并行可视分析流程; 最后通过体网格外表面提取的图元数据轻量化方法，耦合 GPU 真实感绘制， 以支撑实时高质量的仿真设计与分析. 该引擎已集成到国产航空发动机仿真软件中，结果表明，可流畅地支持大规模 仿真应用的设计分析, Trent-1000 发动机模型数千万非结构网格的一键导人交互性能高出 ANSYS 商业软件 8 倍以上.
\end{abstract}

关键词：可视化；计算机辅助工程；高性能计算；实时交互

中图法分类号: TP391.41 DOI: 10.3724/SP.J.1089.2021.19261

\section{Interactive Visual Analysis Engine for High-Performance CAE Simulations}

\author{
Cao Yi ${ }^{1,2)}$, Wang Huawei ${ }^{1,2)}$, Xia Fang ${ }^{1,2)}$, Zhang Zhe ${ }^{2)}$, Ai Zhiwei ${ }^{1,2)}$, Wu Fukun ${ }^{2)}$, and Cao Siming ${ }^{2)}$ \\ 1) (Institute of Applied Physics and Computational Mathematics, Beijing 100094) \\ 2) (Software Center for High Performance Numerical Simulation, China Academy of Engineering Physics, Beijing 100088)
}

\begin{abstract}
Large-scale simulation data poses new challenges to CAE design and analysis. The coherent interaction between pre-processing and post-processing is weak, and the visual interaction of large-scale data is low. Therefore, an interactive visual analysis engine for high-performance CAE simulations is proposed. Firstly, a hybrid data model that efficiently couples geometric models and computational meshes is constructed. Secondly, a parallel visualization pipeline is developed for the coherent interaction of pre-processing and post-processing. Finally, a GPU photorealistic rendering based on lightweight primitives extracted from the external surface of volume meshes is implemented to support real-time high quality CAE design and analysis. The engine has been integrated into aeroengine CAE simulation Apps of China. The results show that it can smoothly support the interactive design and analysis of large-scale simulation applications. Based on this engine, the interactive performance of one-click import and rendering of the Trent-1000 engine model, including tens of millions of unstructured grids, is more than 8 times higher than that of ANSYS software.
\end{abstract}

Key words: visualization; computer aided engineering; high performance computing; real-time interaction

收稿日期: 2021-07-06; 修回日期：2021-08-24. 基金项目：国家重点研发计划(2017YFB0202203)。曹轶(1975一), 男，博士，研 究员, 主要研究方向为科学计算可视化、高性能计算; 王华维(1976-), 男, 博士, 副研究员, 主要研究方向为科学计算可视化、计算 机图形学; 夏芳(1970-), 女, 硕士, 副研究员, 主要研究方向为科学计算可视化; 张哲 (1989-), 男, 硕士, 助理研究员, 主要研究 方向为科学计算可视化; 艾志玮(1973-), 男, 硕士, 副研究员, CCF 会员, 主要研究方向为大规模科学与工程可视化; 吴付坤 (1989-), 男, 博士, 副研究员, 主要研究方向为科学计算可视化; 曹斯铭 (1994一), 女, 博士, 研究实习员, 主要研究方向为科学计 算可视化. 
如果将工业软件比作制造业的皇冠，那么工 业仿真软件就是皇冠上的宝石. 在智能制造的时 代，工业仿真对于新产品的研发是不可或缺的. 当 前，高性能计算技术随着计算机技术的持续发展， 不断被用于解决复杂工程问题, 提升工业仿真设 计的效率. 随着现今产品质量提升以及快速研发 和产品优化需求的爆发式增长, 高性能工业仿真 已经成为工业数字化设计与研发的核心工具.

通过高性能计算机的模拟，高性能仿真应用 可以产生耦合精细几何模型和千万甚至上亿计算 网格单元的大规模仿真数据集, 这对支撑仿真设 计与分析的人机交互力提出新挑战. 例如, 航空发 动机的机匣设计及其包容性分析至关重要, 非包 容性事故会导致机毁人亡的严重空难. 机匣是发 动机叶片的保护罩, 叶片与机匣的间隙不能太小, 否则其热胀冷缩不一致时会导致叶片磨损、起火甚 至断裂; 间隙也不能太大, 否则这样压缩的高压空 气会从间隙回流, 不仅漏气并损失效率, 还可能造 成喘振. 为了获得合理的间隙, 针对机匣、叶片等 几何模型部件的几何形态的交互设计是仿真的重 要环节. 同时, 针对精细离散在计算网格的机匣包 容性仿真结果，支撑大规模仿真结果分析的可视 交互能力不可或缺.

但是，传统科学可视化软件针对模型前处理、 仿真求解、后处理分析的前后连贯交互能力弱. 科 学可视化采用以网格数据为中心的数据模型, 在 其可视化管线中不关注几何模型与计算网格之间 的可持续关联. 为了在可视化管线处理具有连续 型表达的几何模型数据, 需要在预处理过程将连 续几何模型转换为离散化网格数据. 此时, 经转换 的离散网格数据彻底失去了原始几何的描述信息 和模型部件的逻辑关系. 因此, 传统科学可视化软 件无法满足以几何形态牵引的计算机辅助工程 (computer aided engineering, CAE) 仿真设计需求.

另外, 主流 CAE 商业软件对大规模仿真数据 的可视交互能力弱, 虽然其采用几何模型为中心 的数据模型和处理管线, 但仿真交互能力大多仅 能支持上限为数百万的计算网格规模，且仿真交 互的可扩展性受并行核数许可限制. 因此, 商业软 件难以满足具有数千万甚至上亿计算网格规模的 高性能 CAE 仿真需求.

为了突破高性能工业仿真的人机交互瓶颈, 本文提出一个面向高性能工业仿真的交互可视分 析引擎. 首先, 构建一个基于网格片的三维混合数 据模型, 以高效地耦合几何模型和计算网格, 实现
网格数据到几何模型的核心数据模型转变; 其次, 提出面向前后连贯仿真设计的双合约并行可视分 析流程，支撑几何模型与计算网格数据在可视化 管线的可持续关联与高效处理; 最后, 通过体网格 外表体提取的图元数据轻量化方法, 耦合 GPU 实 时真实感绘制, 以支撑大规模仿真数据的实时高 质量分析. 该引擎已集成到国产航空发动机 CAE 仿真应用软件中, 结果表明, 它可流畅地支持大规 模仿真应用的设计分析.

\section{1 相关工作}

工业仿真是智能制造的核心. 当前, 工业设计 与分析领域高度依赖 $\mathrm{ANSYS}^{[1]}, \mathrm{COMSOL}^{[2]}$ 和

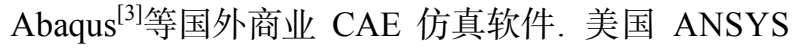
通过技术积累和并购扩张已成为全球 CAE 软件巨 头, 其集结构、流体、电场和磁场分析于一体，应 用横跨石油、能源、军工、汽车和航空多个行业.

ANSYS 等主流商业软件面向仿真全生命周期, 采用几何模型为中心的数据模型, 支撑连贯前后处 理的交互设计分析功能，支持点、边、面、体等几 何形态牵引的全过程人机交互. ANSYS Workbench 包含了高性能计算功能, 但仅支持局部仿真计算, 不支持并行的前后处理任务. ANSYS 并购的美国 EnSight 软件 ${ }^{[4]}$ 可第三方支持仿真并行可视化, 但 其并行核数许可限制了大规模可视化的可扩展性.

另外，传统科学可视化软件面向计算分析过 程, 采用的是以网格数据为中心的数据模型. VTK(visualization toolkit) ${ }^{[5]}$ 是美国 Kitware 公司的 开源可视化库, 它已广泛应用于计算机图形学和数 据可视化等领域. VTK 支持 5 类不同数据格式, 但 是均基于网格数据模型. 美国 VisIt ${ }^{[6]}$ 和 ParaView ${ }^{[7]}$ 等主流开源可视化软件在数据表示层均使用 VTK 网格数据模型, 虽然其数据处理功能丰富, 可扩展 处理大规模数据的能力强, 但是无法支撑几何模 型为中心的 CAE 仿真设计与分析.

可视分析流程 ${ }^{[8]}$ 是科学可视化软件至关重要 的内部机制, 它用于管理数据加载、转换、显示和 保存的全过程, 可描述为一个数据流网络 ${ }^{[9]}$. 可视 计算过程被描述为一系列可执行模块的集合, 各 采用有向图连接, 构成了可视分析流程. 当数据流 经可视分析流程时, 各个模块对数据执行算法运 算, 该有向图表示了数据在各个模块之间的流动 方式. VTK, ParaView 和 VisIt 等科学可视化软件在 数据到图像的转换过程中均采用了可视分析流程. 
显然，基于网格数据模型的可视分析流程仅能够 处理采用网格表达的数据, 无法处理未经网格离 散的几何模型.

按需驱动 ${ }^{[10]}$ 是交互处理大规模科学模拟数据 的有效方式，也是可视分析流程的显著特性 ${ }^{[11]}$. 根据各个模块的处理需求, 仅加载所需要的局部 数据, 有利于提升大规模数据的可视分析性能. 此 外, 科学可视化在使用高性能计算处理大规模数 据集方面具有悠久历史，可视分析流程通常包含 并行计算功能. 通过将大规模数据剖分为一定数 量的子块，采用数据并行模式同时为各个子块数 据复制和执行可视分析流程，可以获得很高的并 行可扩展性. 该处理模式已经成功地应用于当前 的超级计算机上，而主流商业仿真软件不具备上 述大规模处理优势.

针对人机交互的响应度需求, CAE 仿真软件 与科学可视化软件因其任务不同差别较大, 这决 定了适配两者可视交互能力的图形渲染技术. 科 学模拟侧重对批量计算结果的比对和理解，其对 人机交互的响应度要求适中, 并且更加关注能与 海量数据规模相匹配的可扩展并行处理能力. 考 虑多核架构的超算绘制环境，科学可视化一般基 于光栅化的固定渲染流水线 ${ }^{[12]}$. 流水线在内部固 化了一套完整的渲染管线, 只需要在 CPU 代码端 输人渲染所需要的参数并指定特定的开关, 就能 完成不同渲染. 光栅化渲染方法可以多 CPU 高效 地并行加速，但仅支持局部光照 ${ }^{[13]}$ 表现复杂几何 模型. Intel OSPRay 软件 ${ }^{[14]}$ 实现了基于 Intel 处理器 架构加速的光线跟踪渲染方法, 可支持环境光遮 蔽 ${ }^{[15]}$ 等高级光照特效渲染.

CAE 仿真应用关注几何模型的交互设计，对 可视交互的响应度提出了极高要求. 基于多 $\mathrm{CPU}$ 核的并行渲染管线即便非常高效，仍难达到实时. 2001 年, NVIDIA 公司推出了可编程渲染流水线 ${ }^{[16]}$. 基于可编程渲染流水线，应用程序可以自行编写 顶点渲染和像素渲染具体逻辑, 以提升交互绘制 性能，或者获得固定流水线无法渲染的全局光照 特效，表现复杂几何特征. 但是，由于 GPU 的类 CPU 多级访存架构, 其访存性能由寄存器到系统 主存依次变慢. 因此，如何实时交互、可扩展分析 大规模高性能仿真数据, 依然是一项挑战.

\section{2 前后连贯的 CAE 仿真工作流程}

CAE 仿真包括了前处理、仿真求解和后处理 3
阶段流程，如图 1 所示 ${ }^{[17]}$. 其中，前处理阶段负责 定义几何域的形状和拓扑, 设置物理属性和边界 条件定义模拟; 后处理负责仿真结果的可视分析. 上述流程会随产品设计过程而不断迭代. 因此, 在 $\mathrm{CAE}$ 仿真过程中, 特别在前后处理阶段, 保持几 何模型信息的一致连贯传递，对几何模型驱动的 CAE 仿真至关重要, 本文简称为前后连贯. 基于 科学可视化软件支撑高性能 CAE 仿真分析, 前后 连贯则是其中亟待解决的首要问题.

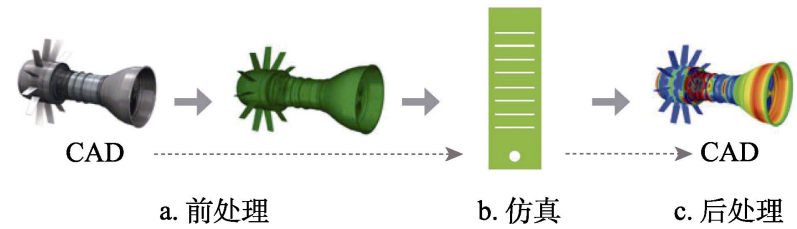

图 1 前后连贯的 $\mathrm{CAE}$ 仿真示意图

\section{3 本文引擎}

为满足几何模型驱动的 $\mathrm{CAE}$ 仿真设计需求, 同时提升大规模仿真数据的交互性能, 本文提出 一个面向高性能工业仿真的交互可视分析引擎. 引擎的 3 层架构如图 2 所示, 包括基于网格片的三 维混合数据模型、面向前后连贯仿真设计的双合约 并行可视分析流程和基于轻量化图元的实时真实 感绘制方法.

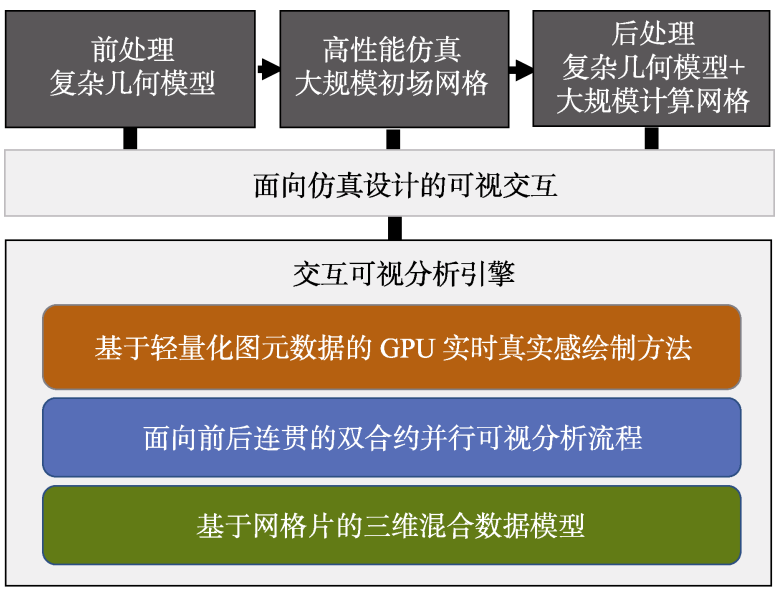

图 2 本文引擎架构

\section{1 基于网格片的三维混合数据模型}

本文提出的高效耦合几何模型和计算网格数 据的三维混合数据模型, 支撑了 “以网格数据为中 心”到“以几何模型为中心”的数据模型转变, 在本 文引擎的数据表示层实现了前后连贯.

本文的三维混合数据模型定义如下。一方面, 混合模型基于几何模型的表面描述定义仿真对象 
的空间轮廓. 几何模型是采用几何概念描述物理 或数学物体形状, 分为线框模型、表面模型和实体 模型. 其中, 表面模型描述了形体表面的所有几何 信息和连接关系, 用于面、边、点的各种几何运算 和集合操作, 它是 CAE 仿真最常用的几何模型. 在实际仿真应用中，一个几何模型可以包含数百 至数千个几何部件.

另一方面，混合模型基于计算网格上的三维体 数据表征仿真计算的离散结果, 并基于网格片进行 仿真数据的组装拼接. 网格片是并行计算的基本调 度单位，是数据存储和管理的核心. 基于网格片的 数据模型, 能显著地提升访存的数据局部性 ${ }^{[18]}$. 如 图 3 所示, 网格片支持多种网格单元, 如结构和非 结构网格等, 其组织拼接可以构成覆盖复杂形状 计算区域的网格块. 采用“网格块-网格片-网格单 元”多层嵌套网格数据结构，可确保并行可视化匹 配高性能计算机多层存储结构.

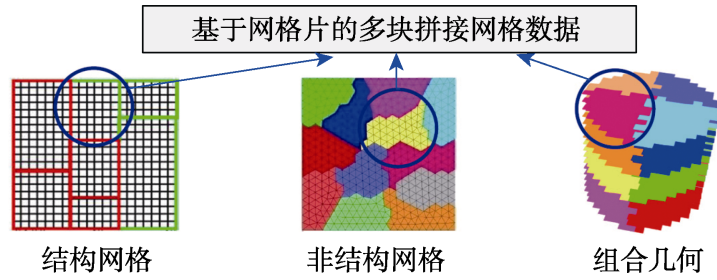

图 3 可匹配高性能计算机的多块拼接网格片数据

特别地，混合数据模型针对几何模型与计算 网格的耦合，采用“计算网格与几何部件”“几何部 件与点、边、面、体等几何信息”的双层耦合连接 方法, 如图 4 所示. 首先, 该混合数据模型将几何 部件的实体编号作为计算网格的一种属性表征, 实现了“几何部件-计算网格”层次的耦合连接. 其 次, 以几何部件为单位在表面网格上进行点、边、 面、体等基本几何信息的离散. 几何信息编号作为

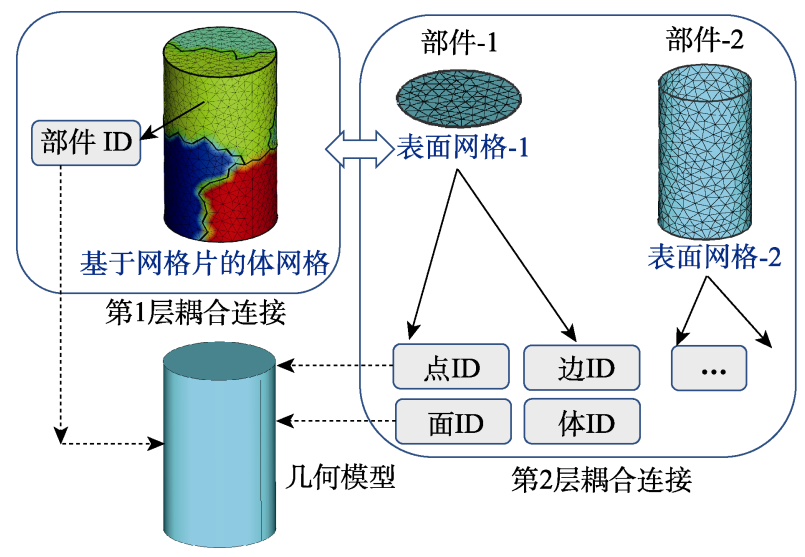

图 4 基于网格片的混合数据模型
几何部件的表面网格的一种属性表征，实现“几何 部件-几何信息”层次的耦合连接.

实际应用的仿真任务通常仅会涉及几何模型 的局部几何部件, 部件数量有限. 为此, 本文数据 模型采用仅对需要仿真分析的几何部件进行按需 网格离散的策略, 可以避免点、边、面、体等几何 信息在三维体网格上的全面离散, 大幅度降低模 型数据存储占用.

\section{2 面向前后连贯设计的双合约可视分析流程}

在高性能仿真的大规模数据作用下，在可视化 管线的数据读人和数据处理阶段, 均呈现数据密集 型和计算密集型的典型特征，构成影响人机交互性 能的核心因素. 科学可视分析引擎 TeraVAP ${ }^{[19]}$ 采用 双合约机制优化可视化管线, 可驱动大规模数据 按需读人和处理. 合约是一种包含空间范围、变量 等元数据信息的数据结构, 它在可视化管线中流 动传递, 并在可视化管线执行前汇总管线中各过 滤器所需的数据和操作模式, 实现按需驱动.

在面向科学数据可视分析流程基础上, 本文 设计了适配复杂几何模型的双合约可视分析流程, 在本文引擎的数据处理层实现前后连贯. 它具有 几何模型直接解析和复杂模型按需离散 2 个显著 特点, 可以避免增量生成大规模的中间数据, 从而 优化几何模型的可视化管线性能.

首先，引擎构造了几何模型元数据，在数据表 示层实现复杂几何模型与双合约可视分析流程的 匹配. 元数据信息包括双合约机制所需的几何部 件空间包围盒、全局区间树和几何属性等. 在构造 几何模型的元数据过程中, 本文的交互引擎采用 边界表示法(B-Rep)表征几何模型.

其次，引擎重构了双合约可视分析流程，支撑 复杂模型的按需离散和高效数据处理. 重构核心 是合约机制. 如图 5 所示，重构的合约实现了基于 边界表示法的数据预篮选, 支撑按需驱动的可视 分析流程. 在流程上行阶段, 几何模型离散展开 前, 基于合约预篮选出分析必需的几何体, 大幅度 降低下行阶段需要离散展开的模型网格数据量;

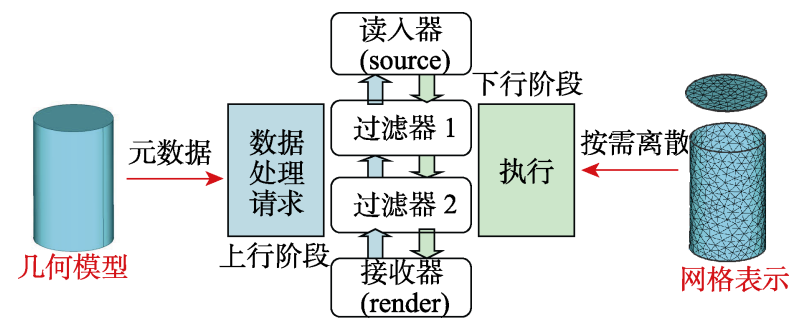

图 5 面向前后连贯仿真设计的双合约可视分析流程 
在流程下行阶段, 重构数据操作算法, 数据操作仅 对实际变化的几何体生成中间数据, 其他未经操作 改变的几何体无须展开, 以确保高效的流程处理.

基于消息传递的整体同步并行(bulk synchronous parallel, BSP) ${ }^{[20]}$ 模型, 引擎可实现高效处理 大规模仿真数据的并行可视分析流程. 基于网格 片数据, 所有进程并行执行局部计算. 这包括本地 计算、全局通信和同步 3 个阶段. 网格片数据局部 性确保了可视分析流程获取数据并行的高效性能.

\section{3 基于轻量化图元数据的实时真实感绘制}

人机交互渲染阶段，引擎实现了基于轻量化 图元数据的 GPU 实时真实感绘制方法, 用以支撑 大规模高性能仿真数据的实时交互和高质量绘制.

(1) 轻量化的图元数据生成

工业仿真应用经常使用模型的外表面作为默 认渲染对象, 方便进行结构强度等仿真结果分析. 而这些仿真应用结果通常是三维体网格数据, 包 含大量无需渲染的体网格内表面, 会引发内存和 性能开销. 为此, 本文实现了一种如图 6 所示有效 提取体网格外表面的数据轻量化方法, 其基本思 想是统计每个体网格面被体网格单元的使用次数. 如果使用 2 次, 表示是内表面, 因为该面是 2 个不 同单元的邻接面; 如果仅使用 1 次，表示是外表 面. 基于表面顶点索引的哈希算法，引擎实现了外 表面快速提取计算.

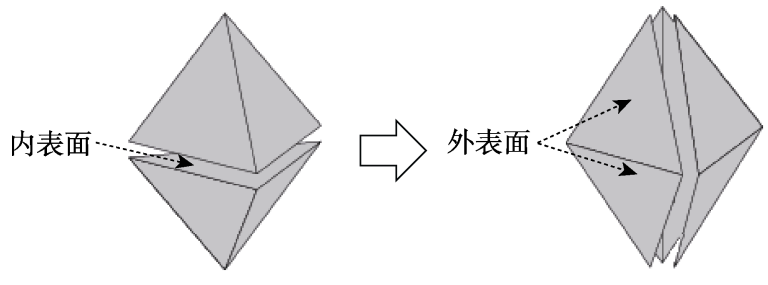

a. 2 个相邻的体网格单元

b. 体网格的外表面提取

图 6 体网格外表面提取的示意图

(2) GPU 实时真实感绘制

科学可视化一般采用光栅化的固定渲染流水 线，它适用于基于多 CPU 核可扩展绘制大规模数 据，但难以实现实时高质量渲染. 本文的交互可视 分析引擎采用了 GPU 可编程渲染流水线, 避免每 帧绘制从 CPU 端到 GPU 端大规模图元数据迁移所 引发的交互性能瓶颈，实现绘制方法与 GPU 硬件 多级访存架构的匹配. 另外, 引擎实现了多材质和 基于环境光遮蔽的实时阴影算法，在模型的禇皱、 孔洞和凹凸位置提供遮挡漫反射光线的真实感特 效, 提升渲染层次感和细节质量, 助力仿真设计.

\section{4 实验测试与分析}

通过前处理和后处理 2 类任务, 测试本文的交 互可视分析引擎针对几何模型、耦合几何模型和计 算网格数据的混合数据的前后连贯交互处理能力, 验证可视交互性能. 本文测试分别使用到配置 24 核处理器、512 GB 内存、NVIDIA Quadro P6000 双显卡的浪潮服务器单节点, 以及用于商业软件 对比测试的配置 Intel Xeon 处理器和 NVIDIA Quadro RTX 5000 显卡的 PC 机 2 种计算环境.

\section{1 前处理}

前处理测试采用 3 种不同规模的仿真模型，验 证交互引擎的前处理支撑能力, 涉及面向前后连 贯仿真设计的双合约可视分析流程和交互绘制方 法. 表 1 所示为模型的特征描述，图 7 所示为各模 型的可视绘制结果. Trent-1000 是英国罗罗公司 (Rolls-Royce)研制的大涵道比民用浴扇发动机; Blade 单叶片模型是航发强度软件的典型应用算例; SIP 电子学器件模型是电磁模拟软件 JEMS-CDS 的 典型应用算例.

表 1 几何模型特征描述

\begin{tabular}{lcrrlr}
\hline 模型名称 & 数据量 $/ \mathrm{MB}$ & 体数 & 面数 & 边数 & \multicolumn{1}{c}{ 点数 } \\
\hline Trent-1000 & 161.9 & 90 & 16641 & 43178 & 28131 \\
Blade & 6.7 & 1 & 526 & 1704 & 1165 \\
SIP & 189.0 & 5145 & 45952 & 94069 & 67031 \\
\hline
\end{tabular}

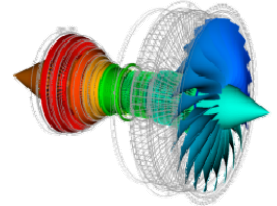

a. Trent-1000

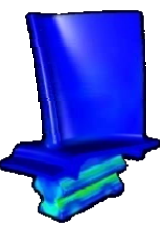

b. Blade

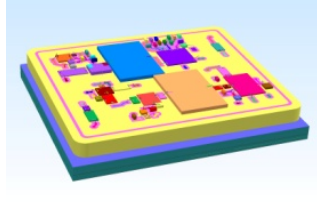

c. SIP
图 7 几何模型的引擎可视化结果

前处理交互操作包括可视交互和几何拾取. 图 7 所示为本文面向几何模型的可视分析流程生 成的图元数据, 并由 GPU 渲染获得的可视化结果. 图 7a 所示为基于几何部件编号在可视分析流程中 执行篮选操作，获得发动机机匣部位并对它进行 几何边的绘制结果, 其显示了引擎可视分析流程 对几何模型处理的匹配性.

可视交互性能取决于几何模型的网格离散数 量; 几何拾取的性能取决于模型中的部件数量. 表 2 所示为传统科学引擎 TeraVAP ${ }^{[19]}$ 与本文交互 引擎的交互性能对比. 可见，科学引擎的固定渲 染流水线显著受限于 $\mathrm{CPU}$ 至 GPU 图元数据迁移 延迟，单帧绘制时间无法实现实时交互. 交互引 
擎解决了上述瓶颈，交互绘制性能提升了 2 3 个 数量级.

表 2 几何模型的可视交互性能

\begin{tabular}{|c|c|c|c|c|}
\hline \multirow{2}{*}{ 模型名称 } & \multicolumn{2}{|c|}{ 离散网格 } & \multicolumn{2}{|c|}{ 单帧绘制时间/s } \\
\hline & 类型 & $10^{-4} \times$ 数量 & 传统 ${ }^{[19]}$ & 本文 \\
\hline Trent-1000 & 四面体 & 8000 & 25.000 & 0.013 \\
\hline Blade & 四面体 & 1995 & 0.420 & 0.003 \\
\hline SIP & 三角形 & 199 & 1.000 & 0.003 \\
\hline
\end{tabular}

表 3 所示为针对包含 5145 个部件的 SIP 模型, ANSYS 软件与本文交互引擎的几何拾取性能对 比. 几何拾取性能取决于几何部件数量和模型的 网格离散规模. 表 3 第 3 行的本文交互引擎是基 于可视分析流程构建区间树的 CPU 加速拾取性 能; 第 4 行的本文交互引擎-1 则采用 CUDA 硬件 加速拾取性能. 显然, 交互引擎的拾取性能优于 ANSYS.

表 3 SIP 模型的交互拾取单帧绘制时间对比 $\mathrm{s}$

\begin{tabular}{lrrrr}
\hline \multirow{2}{*}{ 软件名称 } & \multicolumn{4}{c}{ 几何形状 } \\
\cline { 2 - 5 } & \multicolumn{1}{c}{ 点 } & \multicolumn{1}{c}{ 边 } & \multicolumn{1}{c}{ 面 } & \multicolumn{1}{c}{ 体 } \\
\hline ANSYS $^{[1]}$ & 1.600 & 1.200 & 1.000 & 1.000 \\
传统引擎 $^{[19]}$ & 140.000 & 126.000 & 157.000 & 150.000 \\
本文交互引擎 & 0.523 & 0.568 & 0.548 & 0.651 \\
本文交互引擎-1 & 0.007 & 0.007 & 0.007 & 0.008 \\
\hline
\end{tabular}

\section{2 后处理}

后处理测试使用 Trent-1000 整机模型和航发 燃烧室 2 个仿真算例, 验证交互引擎的后处理支撑 能力. 其中涉及三维混合数据模型、面向前后连贯 仿真设计的可视分析流程以及交互绘制方法.

燃烧室也是航空发动机一个挑战性设计问题. 优秀的燃烧室设计要求均匀混合、均匀燃烧，属于 高温条件下的复杂流动问题. 图 8 所示为航发燃烧 室燃烧分析算例, 它包含 2080 万六面体和四面体 混合网格, 单时刻仿真数据量达到 $12 \mathrm{~GB}$. 一方面, 基于本文的三维混合数据模型, 实现了燃烧室的 几何模型在可视分析流程中与仿真计算网格保持 可持续关联，在后处理阶段支撑了几何模型驱动 的仿真结果设计分析. 另一方面，该三维混合模型 采用以网格片为核心的设计, 可匹配高性能计算 机实现高效并行可视分析. 图 $8 \mathrm{~b}$ 所示为多网格片 的分布情况，同种颜色区域代表一个网格片.

表 4 所示为基于本文算法的 3 个大规模仿真算 例的图元轻量化结果. 3 个算例均采用了六面体或 四面体的计算网格, 经过轻量化产生了四边形或 三角形的面片数据, 相对于原始体网格的面片总

数, 面片约减率平均可达 $90 \%$ 以上. 图 9 所示为 Trent-1000 叶片强度仿真分析结果, 它使用了合金 材质和基于环境光遮蔽的阴影绘制特效.

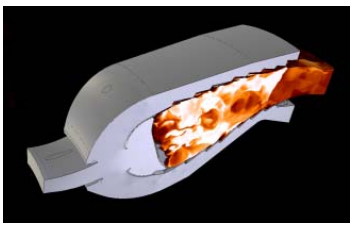

a. 几何构型的仿真结果

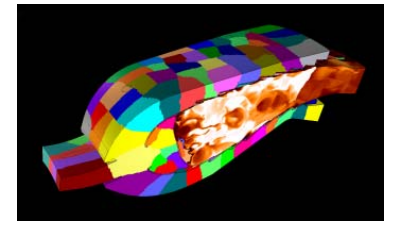

b. 计算网格区域分解

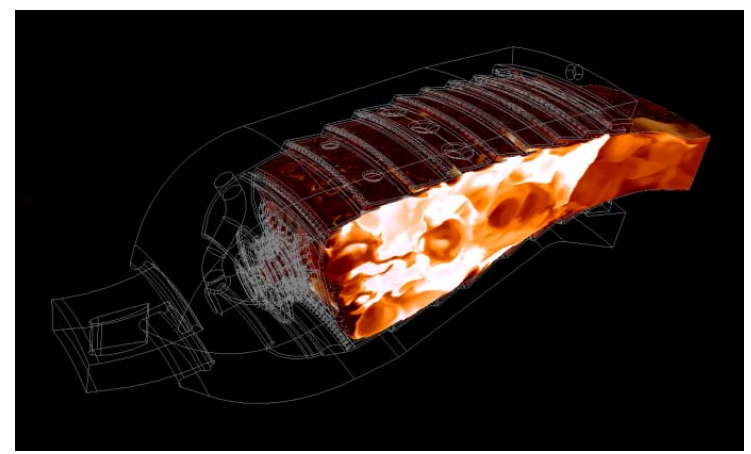

c. 几何线框驱动的仿真设计与分析结果

图 8 面向几何模型的 Combustor 燃烧室仿真设计分析

表 4 仿真算例的图元轻量化结果

\begin{tabular}{lcccccc}
\hline \multirow{2}{*}{ 算例名称 } & \multicolumn{2}{c}{ 网格 } & & \multicolumn{2}{c}{ 图元 } & 面片约 \\
\cline { 2 - 3 } & $10^{-4} \times$ 规模 & 类型 & & $10^{-4} \times$ 规模 & 类型 & 减率 $/ \%$ \\
\hline Trent-1000 & 335 & 六面体 & 112 & 四边形 & 94.4 \\
Blade & 1195 & 四面体 & 52 & 三角形 & 98.9 \\
Combustor & 2080 & 六面体 & 142 & 四边形 & 98.9 \\
\hline
\end{tabular}

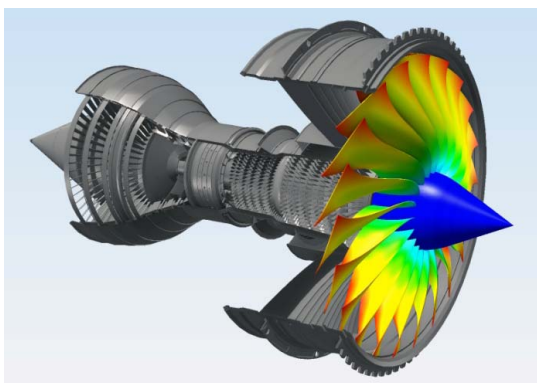

图 9 Trent-1000 航空发动机的涡轮叶片强度仿真分析

\section{3 商业软件交互性能对比}

本文采用计算环境 2(个人计算机)测试主流 CAE 商业软件 ANSYS, COMSOL 和 Abaqus. 其中, 商业软件均在 Windows 7 操作系统下运行, 本文的 交互可视分析引擎在 Centos 7 操作系统下运行.

(1) 渲染效果对比

图 10 所示为 Trent-1000 整机模型的渲染结果 对比. 可以看出, 在材质等高级渲染上, 图 $10 \mathrm{a}$ 的 渲染效果等同于图 $10 \mathrm{~b}$; 在阴影渲染上, 图 $10 \mathrm{a}$ 的 效果优于图 10b, 真实感更强. 


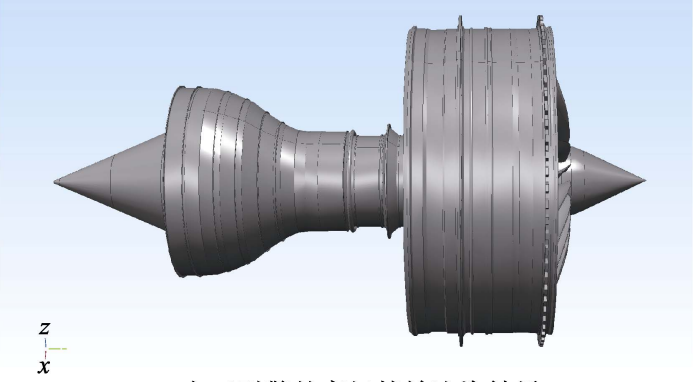

a. 交互引擎的高级特效渲染结果

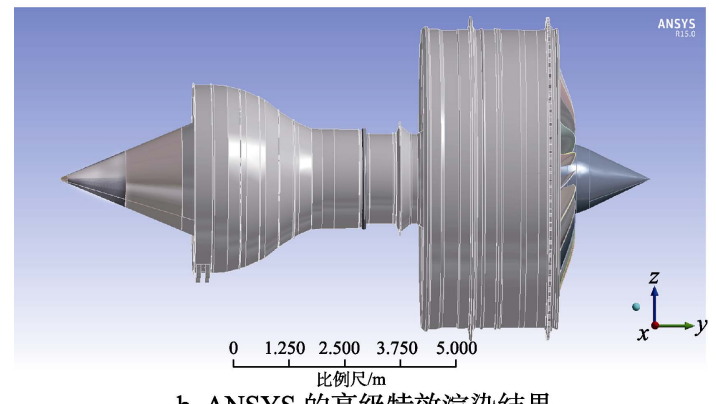

b. ANSYS 的高级特效渲染结果

图 10 Trent-1000 整机模型的渲染效果对比

（2）交互性能对比

表 5 所示为 Trent-1000 整机模型 450 万和 2000 万 2 种规模非结构网格算例的实时交互性能 对比. 分析可见, 针对百万网格规模数据, 交互引 擎和商业软件均交互流畅. 针对千万网格规模数 据，仅交互引擎和 ANSYS 可实现流畅交互.

表 5 CAE 商业软件的交互性能单帧绘制时间对比 $\mathrm{s}$

\begin{tabular}{lcc}
\hline \multirow{2}{*}{ 软件名称 } & \multicolumn{2}{c}{$10^{-4} \times$ 离散网格规模 } \\
\cline { 2 - 3 } & 450 & 2000 \\
\hline 本文交互引擎 & 0.016 & 0.017 \\
$\mathrm{ANSYS}^{[1]}$ & 0.017 & 0.017 \\
$\mathrm{COMSOL}^{[2]}$ & 0.030 & 失败 \\
$\mathrm{Abaqus}^{[3]}$ & 0.024 & 0.200 \\
\hline
\end{tabular}

表 6 和表 7 所示为 Trent-1000 整机模型数千万 非结构网格算例的交互性能对比. 其中，8000 万 网格规模算例采用了浪潮服务器单节点进行测试. 分析可知，由于 ANSYS 不支持网格数据的并行读 取和处理，因此一键文件导人绘图的时间远多于 本文的交互引擎. 分析可得，交互引擎的一键导人 绘制性能高出 ANSYS 软件 8 倍以上.

表 6 Trent-1000 整机 2000 万非结构网格算例对比

\begin{tabular}{cccc}
\hline \multirow{2}{*}{ 软件名称 } & \multicolumn{2}{c}{ 单帧绘制时间 $/ \mathrm{s}$} & \multirow{2}{*}{ 核数 } \\
\cline { 2 - 3 } & 一键导人绘图 & 交互绘制 & \\
\hline 本文交互引擎 & 40.0 & 0.017 & 24 \\
ANSYS WorkBench & 354.0 & 0.019 & 1 \\
\hline
\end{tabular}

表 7 Trent-1000 整机 8000 万非结构网格算例对比

\begin{tabular}{cccc}
\hline \multirow{2}{*}{ 软件名称 } & \multicolumn{2}{c}{ 单帧绘制时间 $/ \mathrm{s}$} & \multirow{2}{*}{ 核数 } \\
\cline { 2 - 3 } & 一键导人绘图 & 交互绘制 nn \\
\hline 本文交互引擎 & 96.0 & 0.01 & 16 \\
ANSYS APDL & 736.2 & 22.00 & 1 \\
\hline
\end{tabular}

\section{5 结 语}

本文提出一个交互可视分析引擎，它从数据 表示模型、可视分析流程和图元绘制方法 3 个层次 解决大规模仿真数据对仿真设计分析造成的交互 力瓶颈问题，可以流畅地支持基于几何模型的大 规模仿真应用的设计分析, Trent-1000 发动机模型 算例一键导人交互性能高出 ANSYS 商业软件 8 倍 以上. 当前, 交互引擎是由科学可视化引擎发展而 来, 它虽然能可扩展处理大规模高性能仿真数据, 但是一键导人绘图的交互性能仍难以达到实时, 无法支撑时序仿真数据集分析. 未来, 需探讨数据 轻量化、多分辨率技术与引擎数据模型等的结合, 进一步优化交互性能.

\section{参考文献(References):}

[1] Thompson M K, Thompson J M. ANSYS mechanical APDL for finite element analysis[M]. Oxford: Butterworth-Heinemann, 2017

[2] Freels J D, Arimilli R, Bodey I T. Exploiting new features of COMSOL version 4 on conjugate heat transfer problems[OL]. [2021-07-06]. https://www.researchgate.net/publication/323401471_Exploiting_New_Features_of_COMSOL_Version_4_on_Conjugate_Heat_Transfer_Problems

[3] Ciesielska-Wrobel I. Finite element modeling of textiles in Abaqus ${ }^{\mathrm{TM}}$ CAE[M]. Boca Raton: CRC Press, 2019

[4] Frank R, Krogh M F. The EnSight visualization application[M] //High Performance Visualization Enabling Extreme-Scale Scientific Insight. New York: Chapman and Hall/CRC Press, 2012: 429-442

[5] Schroeder W J, Martin K M, Lorensen B. The visualization toolkit: an object oriented approach to 3D graphics[M]. 4th ed. New York: Kitware Inc., 2006

[6] LLNL. VisIt user's manual[OL]. [2021-07-06]. https:// visit-sphinx-github-user-manual.readthedocs.io/en/develop/

[7] Ayachit U. The ParaView guide: a parallel visualization application[M]. New York: Kitware Inc., 2004

[8] Lucas B, Abram G D, Collins N S, et al. An architecture for a scientific visualization system[C] //Proceedings of the $3 \mathrm{rd}$ Conference on Visualization. Los Alamitos: IEEE Computer Society Press, 1992: 107-114

[9] Schroeder W J, Martin K M, Lorensen W E. The design and implementation of an object-oriented toolkit for 3D graphics 
and visualization[C] //Proceedings of the 7th Annual IEEE Visualization. Los Alamitos: IEEE Computer Society Press, 1996: 93-100

[10] Hadwiger M, Beyer J, Jeong W K, et al. Interactive volume exploration of petascale microscopy data streams using a visualization-driven virtual memory approach[J]. IEEE Transactions on Visualization and Computer Graphics, 2012, 18(12): 2285-2294

[11] Moreland K. A survey of visualization pipelines[J]. IEEE Transactions on Visualizations and Computer Graphics, 2013, 19(3): 367-378

[12] Akenine-Möller T, Haines E. Real-time rendering[M]. 2nd ed. Boca Raton: A K Peters/CRC Press, 2002

[13] Akenine-Möller T, Haines E, Hoffman N, et al. Real-Time Rendering[M]. 4rd ed. Boca Raton: A K Peters/CRC Press, 2018

[14] Wald I, Johnson G P, Amstutz J, et al. OSPRay: a CPU ray tracing framework for scientific visualization[J]. IEEE Transactions on Visualization and Computer Graphics, 2017, 23(1):
931-940

[15] Suffern K. Ambient occlusion[M] //Suffern K. Ray Tracing from the Ground Up. Boca Raton: A K Peters/CRC Press, 2016: 309-324

[16] Kessenich J, Baldwin D, Rost R. The OpenGL ${ }^{\circledR}$ shading language[OL]. [2021-07-06]. https://www.khronos.org/registry/ OpenGL/specs/gl/GLSLangSpec.4.10.pdf

[17] NVIDIA. NVIDIA Quadro GP100 bring optimal designs to market faster[OL]. [2021-07-06]. https://images.nvidia.com/ content/pdf/quadro/product-solutions/quadro-gp100-cae-solutionsheet-385756-20170615-nv-us-lr.pdf

[18] Mo Z Y, Zhang A Q, Cao X L, et al. JASMIN: a parallel software infrastructure for scientific computing $[\mathrm{J}]$. Frontiers of Computer Science in China, 2010, 4(4): 480-488

[19] Cao Y, Mo Z Y, Ai Z W, et al. An efficient and visually accurate multi-field visualization framework for high-resolution climate data[J]. Journal of Visualization, 2016, 19(3): 447-460

[20] Gerbessiotis A V, Siniolakis C J. Merging on the BSP model[J]. Parallel Computing, 2001, 27(6): 809-822 